\title{
Three independent genetic profiles based on mucin expression in early differentiated-type gastric cancers-a new concept of genetic carcinogenesis of early differentiated-type adenocarcinomas
}

\author{
Tamotsu Sugai ${ }^{1}$, Wataru Habano ${ }^{2}$, Noriyuki Uesugi ${ }^{1}$, Yu-Fei Jao ${ }^{1}$, Shin-ichi Nakamura ${ }^{1}$,
} Kaoru Abe $^{3}$, Akinori Takagane ${ }^{3}$ and Masanori Terashima ${ }^{4}$

${ }^{1}$ Division of Pathology, Central Clinical Laboratory, Iwate Medical University, Morioka, Japan; ${ }^{2}$ DNA Laboratory, Iwate Medical University, Morioka, Japan; ${ }^{3}$ First Department of Surgery, School of Medicine, Iwate Medical University, Morioka, Japan and ${ }^{4}$ First Department of Surgery, School of Medicine, Fukushima Prefectural Medical College, Fukushima, Japan

\begin{abstract}
Recent molecular studies have shown that the genetic profiles of differentiated-type adenocarcinomas of the stomach are associated with distinct cellular mucin phenotypes (gastric- intestinal- and mixed-phenotypes). Therefore, we examined whether these cellular mucin phenotypes reflect specific molecular genetic alterations, and whether the phenotypes can be used to help categorize the intramucosal neoplasias of gastric tumors. We subclassified tumors into four cellular phenotypes using immunohistochemical mucin analysis. In all, 62 early gastric carcinomas (gastric-phenotype, 13; intestinal-phenotype, 17; mixed-phenotype, 31; unclassifiedphenotype, 1) were examined using a combination of polymerase chain reaction microsatellite assays and immunohistochemical analysis in order to detect chromosomal allelic losses of multiple cancer-related chromosomal loci (1p, 3p, 4p, 5q, 8p, 9p, 13p, 17p, 18q and 22q), microsatellite instability (MSI), and overexpression of the p53 protein. In addition, we analyzed the relationship between MSI status and hMLH1 promoter hypermethylation, which is thought to be a cause of high MSI status. For gastric phenotype cancers, the frequency of $3 p$ allelic loss was higher than that of other microsatellite markers, whereas $5 q$ allelic loss was frequently found in intestinal phenotype cancers. The genetic profile of mixed phenotype cancers is comprised of two distinct genetic types: LOH and MSI types. In the former, 5q, 3p and 18q allelic losses are seen frequently in intramucosal carcinomas. On the other hand, $17 p, 1 p$ and $9 p$ allelic losses are associated with the development of submucosal carcinomas. MSI was observed only in mixed phenotype cancers (six of 31 mixed phenotype cancers). Overexpression of the $\mathbf{p} 53$ protein is common in differentiated-type gastric cancers. In addition, the MSI status of the tumor cells was correlated with the extent of hypermethylation of the hMLH1 promoter. We suggest that the cellular mucin phenotypes of the differentiated-type adenocarcinomas result from distinct genetic alterations.
\end{abstract}

Modern Pathology (2004) 17, 1223-1234, advance online publication, 21 May 2004; doi:10.1038/modpathol.3800170

Keywords: early gastric cancer; loss of heterozygosity; microsatellite instability; mucin expression

\section{Introduction}

Gastric adenocarcinomas are classified into intestinal- and diffuse-types. ${ }^{1}$ Intestinal-type cancers demonstrate biological and epidemiological features

Correspondence: Dr T Sugai, MD, Division of Pathology, Central Clinical Laboratory, Iwate Medical University, 19-1, Morioka 020-8505, Japan.

E-mail: tsugai@cocoa.ocn.ne.jp

Received 9 January 2004; revised 26 March 2004; accepted 28 March 2004; published online 21 May 2004 that are distinct from those of diffuse-type cancers. ${ }^{1,2}$ These data suggest that tumors displaying different histological types are associated with distinct biological behaviors. ${ }^{1-3}$ For example, intestinal-type carcinomas tend to metastasize to the liver, whereas diffuse-type tumors disseminate to the peritoneum. ${ }^{1-3}$ In addition, it is widely accepted that intestinal-type carcinomas are genetically distinct from diffuse-type carcinomas. ${ }^{3,4}$ Whereas intestinal-type carcinomas frequently show mutations of the p53 gene and extensive loss of heterozygosity $(\mathrm{LOH})$ of cancer-related genes., ${ }^{4,5}$ diffuse-type 
carcinomas demonstrate mutations of the $p 53$ gene only infrequently and have a low frequency of $\mathrm{LOH}^{4,5}$ The current categorization is useful for understanding morphological changes, histogenesis and biological features of gastric cancers.

Recent studies have suggested that intestinal-type adenocarcinomas (differentiated-type carcinomas), which have a glandular structure, may be subclassified into three groups based on their mucin phenotype: foveolar-, intestinal- and combined types. ${ }^{6,7}$ This phenotypical classification using mucin-based immunohistochemical procedures represents genetically different subtypes. ${ }^{6,7}$ Recent studies have shown that the genetic backgrounds of differentiated-type carcinomas differ among mucin phenotypes. ${ }^{6,7}$ For example, Endoh et $a l^{7}$ have demonstrated that microsatellite instability (MSI) status, which represents mutations of short-tandem repeat sequences distributed throughout the genome, is closely related to expression of the foveolar mucin phenotype. ${ }^{4}$ Classification by phenotype may allow us to understand the genetic basis of carcinogenesis of the differentiated-type adenocarcinomas and may play a role in tumor diagnosis.

The multistep carcinogenesis model established by Vogelstein et $a l^{8}$ for colorectal tumors has not been confirmed for gastric cancers. Recently, two types of genetic instabilities have been described in human tumors: chromosomal instability (CIN) and MSI. ${ }^{9}$ The former is characterized by tumor cell $\mathrm{LOH}$, and as well as by mutations of the ki-ras and p53 genes in colorectal carcinomas. ${ }^{9}$ The latter type (MSI) has specific genetic alterations that are different from LOH, and which may be involved in carcinogenesis. ${ }^{10} \mathrm{CIN}$ type (LOH type) is thought to result from the multistep carcinogenesis of tumor cells, that is, the acquisition of stage-specific genetic alterations. Although it seems that this correlation is less strict in gastric cancers than that initially proposed for colorectal tumors, determination of the accumulation pattern of genetic alterations in tumor cells should improve current models of tumor development.

In order to correlate the cellular phenotypes (determined by immunohistochemical mucin analysis) with genetic alterations in differentiated-type adenocarcinomas of the stomach, we studied allelic losses of multiple cancer-related chromosomal loci, p53 overexpression and microsatellite instability (MSI) in these differentiated-type tumors. In addition, we examined the correlation of MSI status with hypermethylation of the $h M L H 1$ promoter, which may cause MSI of tumor cells.

\section{Materials and methods}

\section{Patient Samples}

Samples from 62 patients (40 men, 22 women, mean age 62.6 years, range $43-85$ years) with early gastric adenocarcinomas were analyzed for this study (from the Division of Pathology, Central Clinical Laboratory, Iwate Medical University, Japan). No patients had undergone chemo- or radiotherapy prior to surgery. Patients with a family history or with a past medical history of gastric cancer were not included in the study.

The surgical specimens were fixed in $20 \%$ buffered-formalin and embedded in paraffin wax. All sections were routinely prepared and stained with hematoxylin and eosin (HE). In short, sections were made according to the tumor size. The number of sections varied from 3 to 42 . One of the sections was chosen as a representative sample. This section was obtained from a region of the resected stomach adjacent to the region used for genetic analysis. The histological criteria used to make the histological diagnosis of intramucosal cancer were based on Japanese histological criteria, ${ }^{11}$ which differ from those accepted by Western pathologists who consider invasion into the lamina propria of the mucosa mandatory for the diagnosis of carcinoma, whereas the preferred criteria among Japanese pathologists are nuclear and structural abnormalities (atypia). ${ }^{12}$ Well-differentiated and moderately differentiated tubular adenocarcinomas and papillary adenocarcinomas corresponding to the intestinal-type (Lauren's classification $^{1}$ ) were used to analyze genetic alterations (defined as differentiated-type adenocarcinomas). However, poorly differentiated adenocarcinomas and signet ring cell carcinomas that were classified as diffuse type according to Laurens's classification ${ }^{1}$ were excluded from the study. In addition, cases with a solid pattern of poorly differentiated adenocarcinomas were also omitted. Early cancers were further subdivided into two groups, intramucosal and submucosal cancers, both of which exhibit a potential for lymph node metastasis. ${ }^{13}$

For molecular investigations, tumor tissue was isolated from the resected stomach using biopsy forceps within $30 \mathrm{~min}$ of resection. The most distant normal gastric mucosa from the neoplasm was removed from the submucosa using scissors. Tissue for clinicopathological analysis was obtained from a region of the resected stomach adjacent to the region used for genetic analysis. All of the tissue samples contained cancer tissue. Only tumor samples where the neoplastic cells accounted for at least $50 \%$ of the tissue cell population were selected (eight of 70 tumors were omitted due to failure to meet these criteria).

The DNA was extracted from isolated normal and tumor tissue by sodium dodecyl sulfate (SDS) lysis and proteinase $\mathrm{K}$ digestion, followed by a phenolchloroform procedure that was reported previously. ${ }^{14}$

\section{Immunohistochemical Studies}

Expression of MUC 2 (Novocastra Laboratories, Newcastle, UK), CD10 (Novocastra Laboratories), 
MUC5AC (Novocastra Laboratories) and HIK1083 (M-GGMC-1, Kanto Chemical Co., Tokyo, Japan) was studied using the DAKO Envision + system (dextran polymers conjugated with horseradish peroxidase, DAKO, Copenhagen, Denmark), as previously described. ${ }^{15}$ In addition, expression of p53 protein was examined using a monoclonal antibody to the p53 protein (DO7, DAKO, Copenhagen, Denmark). Paraffin-embedded tissue blocks were cut in $5-\mu \mathrm{M}$ sections, which were deparaffinized in xylene and then rehydrated in alcohol with distilled water. Endogenous peroxidase activity was inhibited by incubating the sections in $3 \% \mathrm{H}_{2} \mathrm{O}_{2}$ for $20 \mathrm{~min}$. Antigen retrieval was required for all antibodies and consisted of microwave treatment of sections for 20 min in citrate buffer ( $\mathrm{pH}$ 7.0) in a microwave oven at $300 \mathrm{~W}$ (H2500, Microwave Processor, Bio Rad, USA) as previously described. ${ }^{16}$ Endogenous biotin activity was blocked using a DAKO Envision+ system that prevents nonspecific background staining. Sections were then counterstained with hematoxylin and were independently evaluated by two experienced pathologists (ST and NS). In some cases, in which the evaluation led to different results, a consensus interpretation was reached after re-examination. p53-positive cells showed distinct brown staining of the nuclei with intratumoral heterogeneity. Only nuclear staining was measured as positive, and all stained tumor cell nuclei were regarded as positive. Tumor overexpression of p53 was defined as $>10 \%$ positive cells given that overexpression of p53 that was defined as $>10 \%$ of tumor cells was relatively related to p53 gene mutation (exons 5-8) in colorectal carcinomas (data not shown).

\section{Evaluation of Mucin Expression}

Early gastric cancers were classified into four groups according to their immunostaining pattern. Gastric phenotype cancer was defined as tumors with predominantly intracytoplasmic mucin of the gastric phenotype, as determined by immunostaining of human gastric mucin (MUC5AC) and/or of pyloric gland mucin (HIK1083), but without MUC-2-positive intestinal-type goblet cells. Intestinal phenotype cancer refers to a tumor with MUC2-positive cells and/or CD10-positive cells (along with a brush border); these were recognizable as mature neoplastic cells with features of small intestinal absorptive cells. Mixed-type cancer was defined as a tumor with immunostaining that was consistent with both gastric and intestinal phenotypes. Finally, tumors without positive immunostaining consistent with either gastric or intestinal phenotypes were referred to as having an unclassified phenotype. If more than $5 \%$ of the tumor cells were immunopositive, the tumor was regarded as positive for that antibody; otherwise, it was classified as negative, according to previous reports. ${ }^{16,17}$
Analysis of Allelic Losses by Polymerase Chain Reaction

Allelic losses on chromosomes 1p, 3p, 4p, 5q, 8p, $9 p, 13 q, 17 p, 18 p$ and $22 q$ were examined in paired tumor and normal DNA samples obtained from 62 early gastric cancer patients using 22 highly pleomorphic microsatellite markers (D1S228, D1S548, D3S2402, D3S1234, D4S2639, D4S1601, D5S107, D5S346, D5S299, D5S82, D8S201, D8S513, D8S532, D9S171, D9S1118, D13S162, TP53, D18S487, D18S34, D22S274, D22S1140 and D22S1168). These microsatellite markers have been reported frequently in gastric $^{18}$ or colorectal carcinomas. ${ }^{19,20}$ Microsatellite markers on $1 p, 8 p$ and $22 q$ that are used for analysis of $\mathrm{LOH}$ in colorectal carcinomas were examined, since the histological appearance of differentiated-type carcinoma of the stomach resembles that of colorectal carcinomas. In addition, a variable number of tandem repeat polymorphisms at the DCC locus were tested. Microsatellite sequences were obtained from specific primers reported in the Genome Database (http://gdbwww.gdb.org/gdb/).

PCR reactions were performed using a thermal cycler (GeneAmp PCR System 9600, Perkin-Elmer, CA, USA) with 50-100 ng of genomic DNA as template, $25 \mathrm{pM}$ of each primer, $0.2 \mathrm{mM}$ deoxynucleotide triphosphate $(\mathrm{dNTP}), 1 \times$ reaction buffer containing $1.5 \mathrm{mM} \mathrm{MgCl}_{2}$, and $1.5 \mathrm{U}$ Taq polymerase (Boehringer Mannheim Co., Germany) in a final reaction volume of $25 \mu \mathrm{l}$. Samples were processed for 25-30 cycles, with each cycle consisting of $30 \mathrm{~s}$ at $94^{\circ} \mathrm{C}, 1 \mathrm{~min}$ at $55-58^{\circ} \mathrm{C}$, and $2 \mathrm{~min}$ at $72^{\circ} \mathrm{C}$, followed by a final $10 \mathrm{~min}$ extension at $72^{\circ} \mathrm{C}$. For quantitative detection of the allelic loss at each locus, PCR-LOH (loss of heterozygosity) analysis was performed as described previously. ${ }^{19,20}$ A $1-\mu$ l aliquot of the PCR product was added to 3- $\mu$ l formamide and a 0.5- $\mu$ l TAMRA 500 size standard (Applied Biosystems, CA, USA), loaded on a $6 \%$ polyacrylamide-8-M urea gel, and run for $2-6 \mathrm{~h}$ in a 373A Automated Sequencer (Applied Biosystems, CA, USA) at a constant power of $30 \mathrm{~W}$.

\section{Determination of Allelic Loss}

The peaks produced by the normal DNA sample were used to determine whether the cancerous sample was homozygous (one peak) or heterozygous (two peaks). The allelic ratio was calculated as described by Habano et al ${ }^{19}$ A tumor was considered to have allelic loss if the allele peak ratio was less than or equal to 0.60 , representing an allelic signal reduction of at least $40 \%$. We interpreted this allelic imbalance as allelic loss with the provision that, in some cases, the changes in the allele peak ratio may have resulted from allelic amplification. Tumors exhibiting MSI at a given locus were not evaluated for allelic loss. The data were collected 
automatically and analyzed using GeneScan software (Applied Biosystems, CA, USA) to determine the allele score and to assess the possibility of allelic loss.

\section{Analysis of Microsatellite Instability}

Three dinucleotide repeat markers (D2S123, D5S346 and D17S250) and two adenine mononucleotide repeats (BAT 25 and BAT26) were used to determine the presence of tumor MSI. MSI-positive status of a tumor was defined as being present when a novel, abnormal-sized band occurred in the tumor sample, as compared to the corresponding normal DNA sample. MSI-positive colorectal carcinomas were used as controls in the study and were divided into two groups, those with high-level instability (ie, MSI at $\geqq 33 \%$ of loci) and those with low-level instability (ie, MSI at $\leqq 17 \%$ of loci), as described previously. ${ }^{20,21}$ However, tumors with only one alteration of the marker examined using the above criteria, and those were previously categorized as MSI low, were considered MSI-negative tumors in this study.

\section{DNA Methylation Analysis}

DNA methylation patterns of the $h M L H 1$ promoter were determined using methylation-specific PCR (MSP), as described previously. ${ }^{22,23}$ MSP distinguishes unmethylated from methylated alleles of a given gene based on sequence changes that are produced following bisulfite treatment of DNA, which converts unmethylated cytosines to uracils while leaving methylated cytosines unaffected. Subsequent polymerase chain reactions (PCRs) using primers specific to sequences corresponding to either methylated or unmethylated DNA were performed. The primer sequences of the methylated and unmethylated regions of the $h M L H 1$ promoter have been described previously. ${ }^{23,24}$ In the present study, two sets of primers (referred to as M1 and M2) were used to identify $h M L H 1$ promoter hypermethylation. Briefly, $1 \mu \mathrm{g}$ of genomic DNA was denatured by treatment with $\mathrm{NaOH}$ and modified by sodium bisulfite. DNA samples were then purified using a Wizard DNA purification resin (Promega, Madison, WI, USA), retreated with $\mathrm{NaOH}$, precipitated with ethanol, and resuspended in water. PCR of modified DNA was carried out as described elsewhere. ${ }^{22,23}$ The PCR products were then loaded onto $3 \%$ agarose gels, stained with ethidium bromide, and visualized under UV illumination. Samples showing signals approximately equivalent to that of the size marker were regarded as methylated. Samples giving faint positive signals were repeated two times and only those samples with consistent positive signals were interpreted as methylated.

\section{Statistical Analysis}

The data were analyzed using the $\chi^{2}$ test with the aid of StatView-IV software (Abacus Concepts, Berkeley, CA, USA). Samples were determined to be significantly different when the $P$-value was less than 0.05 .

\section{Results}

\section{Mucin Phenotypes}

Of the 62 early gastric adenocarcinomas, 17 (27.4\%) showed an intestinal phenotype, defined as a tumor with positive immunostaining with an MUC2 and/ or a CD10 antibody. MUC2 expression was seen within the cytoplasm around the nuclei, whereas CD10 staining was found along the brush border. Both MUC2 and CD10 immunopositivity was observed in 11 of 17 cases (64.7\%). The remaining six tumors expressed MUC2 alone. Of 62 gastric adenocarcinomas, $13(21.0 \%)$ had a gastric phenotype, defined as a tumor with positive immunostaining for MUC5AC and/or HIK1083. Five of 13 tumors expressed both MUC5AC and HIK1083 immunopositivity, whereas eight tumors exhibited MUC5AC immunopositivity alone.

Of 62 gastric adenocarcinomas, 31 (50\%) demonstrated a mixed phenotype, which was defined as a tumor with characteristics of both intestinal and gastric phenotypes. Only one of 62 adenocarcinomas displayed an unclassified phenotype, defined as no expression of markers for intestinal or gastric mucin.

Clinicopathological data for these three mucin phenotypes associated with gastric cancer are shown in Table 1 . In addition, details concerning the antibodies used in this study are listed in Table 2.

\section{Analysis of Allelic Losses on Each Chromosomal Locus and Microsatellite Instability}

To determine the frequencies of $1 p, 3 p, 4 p, 5 q, 8 p$, $9 p, 13 q, 17 p, 18 p$ and $22 q$ allelic losses in differentiated-type cancers, we analyzed 62 paired normal/tumor DNA samples using PCR methods. Allelic loss at each locus was confirmed. Allelic loss at the $3 p$ locus was found frequently in gastricphenotype cancers (5/10, allelic loss/informative case). However, few tumors with the gastric phenotype showed allelic loss at other microsatellite markers (Figure 1). Allelic loss on $3 p$ was found most frequently at D3S1234. In the intestinalphenotype cancers, the frequency of $5 q$ allelic loss $(7 / 14,50 \%)$ was higher than that seen for other microsatellite markers, as shown in Figure 2. In mixed-phenotype cancers, the genetic profile was classified into two genetic categories (Table 3). One was characterized by multiple allelic losses at many chromosomal loci (called LOH type), and the other was of the MSI type. In mixed-phenotype cancers 
Table 1 Clinicopathological feature of early differentiated-type adenocarcinomas according to mucin phenotypes

\begin{tabular}{|c|c|c|c|c|}
\hline & Intestinal-phenotype & Gastric-phenotype & Mixed-Phenotype & Unclassified type \\
\hline Total & 17 & 13 & 31 & 1 \\
\hline Age (years) & $43-80$ & $44-85$ & $41-80$ & 65 \\
\hline $\operatorname{Sex}(M / W)$ & $11 / 6$ & $8 / 5$ & $20 / 11$ & $1 / 0$ \\
\hline Size (mean, mm) & $10-60(22.3)$ & $11-47(17.8)$ & $18-110(34.1)$ & 25 \\
\hline \multicolumn{5}{|l|}{ Location } \\
\hline $\mathrm{L}$ & 8 & 4 & 16 & 1 \\
\hline M & 7 & 7 & 12 & 0 \\
\hline $\mathrm{U}$ & 2 & 2 & 3 & 0 \\
\hline \multicolumn{5}{|l|}{ Macroscopic type } \\
\hline I & 3 & 0 & 3 & 0 \\
\hline IIa & 4 & 3 & 10 & 0 \\
\hline IIc & 10 & 10 & 18 & 1 \\
\hline \multicolumn{5}{|l|}{ Macroscopic type } \\
\hline WDA & 14 & 5 & 19 & 0 \\
\hline MDA & 3 & 8 & 12 & 1 \\
\hline \multicolumn{5}{|l|}{ Depth } \\
\hline $\mathrm{m}$ & 12 & 9 & 15 & 0 \\
\hline $\mathrm{sm}$ & 5 & 4 & 16 & 1 \\
\hline
\end{tabular}

M, man; W, woman; L, lower site; M, middle site; U, upper site; WDA, well-differentiated adenocarcinoma; MDA, moderately differentiated adenocarcinoma; m, mucosa; sm, submucosa.

Table 2 List of antibodies used in the present study

\begin{tabular}{lllll}
\hline & Clone & Dilution & Pretreatment & Source \\
\hline p53 & DO7 & $1: 100$ & Heating & Dako, USA \\
MUC2 & Ccp58 & $1: 100$ & Heating & Navocastra, UK \\
CD10 & 56C6 & $1: 100$ & Heating & Navocastra, UK \\
MUC5AC & CLH2 & $1: 100$ & Heating & Navocastra, UK \\
HIK1083 & MGGMC-1 & $1: 100$ & Heating & Kanto Chemical, \\
& & & & Japan \\
\hline
\end{tabular}

displaying the LOH type, $5 q, 18 q$ and $3 p$ allelic losses were observed frequently in intramucosal cancers, whereas frequent allelic losses at $17 \mathrm{p}, 1 \mathrm{p}$ and $9 p$ loci occurred in submucosal cancers (Table $3)$. The frequency of $17 p$ and $1 p$ allelic losses in intramucosal and submucosal carcinomas was different. Six tumors with a mixed phenotype showed MSI, which was detectable in four of five microsatellite markers that were examined. Although the frequency of MSI was higher in mixed-phenotype tumors than in gastric or intestinal phenotypes, these differences did not reach statistical significance. Tumors were also analyzed by methylationspecific PCR in order to detect hypermethylation of the $h M L H-1$ promoter. Hypermethylation was present in 17 of 62 tumors $(27.4 \%)$, as detected using either M1 or M2 primers. hMLH1 promoter hypermethylation was detected more often in tumors with MSI (5/6 tumors). In addition, there was a significant difference in the frequency of $h M L H 1$ promoter hypermethylation status between MSI $+(5 / 6,83.3 \%)$ and MSI- $(12 / 56$ cases, $20 \%)$ tumors $(P<0.05)$.

In the single tumor with an unclassified type, multiple allelic losses at 3p, 4p, 5q, 9p, 13q, 17p, $18 \mathrm{q}$ and $22 \mathrm{q}$ were detected.

\section{Immunohistochemical Expression of p53}

No p53 immunoreactivity was detected in the nonneoplastic gastric mucosa around the tumors or in the stromal cells within the tumor masses. Intestinal metaplasia, which was a common feature of the adjacent mucosa of the intestinal-type cancers, was also negative for p53 immunoreactivity.

The frequency of p53 overexpression in tumors is shown in Table 4. No significant differences in the frequency of p53 overexpression in gastric-, intestinal- and mixed-phenotype cancers were seen $(5 / 13$, 8/17 and 14/31 cases, respectively). With regards to mixed-phenotype tumors, there was no difference in the frequency of p53 overexpression between intramucosal and submucosal carcinomas $(7 / 15$, and 7/16 cases, respectively). On the other hand, submucosal cancers of the intestinal-phenotype tended to have a higher frequency of p53 positivity (4/5 cases) than intramucosal cancers (4/12 cases), but there was no statistical difference between these.

We next examined the association between p53 overexpression and MSI status. In 31 mixed-phenotype cancers, p53 overexpression was found in four of six tumors with high MSI (66.7\%), as compared to p53 overexpression in 10 of the other 25 tumors $(40.0 \%)$.

Overexpression of the p53 protein was also observed in the one unclassified tumor.

Representative examples of genetic analysis of mixed-phenotype tumors are shown in Figures 3 and 4 . In addition, a representative example of the methylation status of mixed phenotype cancers is shown in Figure 5.

\section{Clinicopathological correlations}

There were no significant differences among clinicopathological features of four mucin phenotypes 


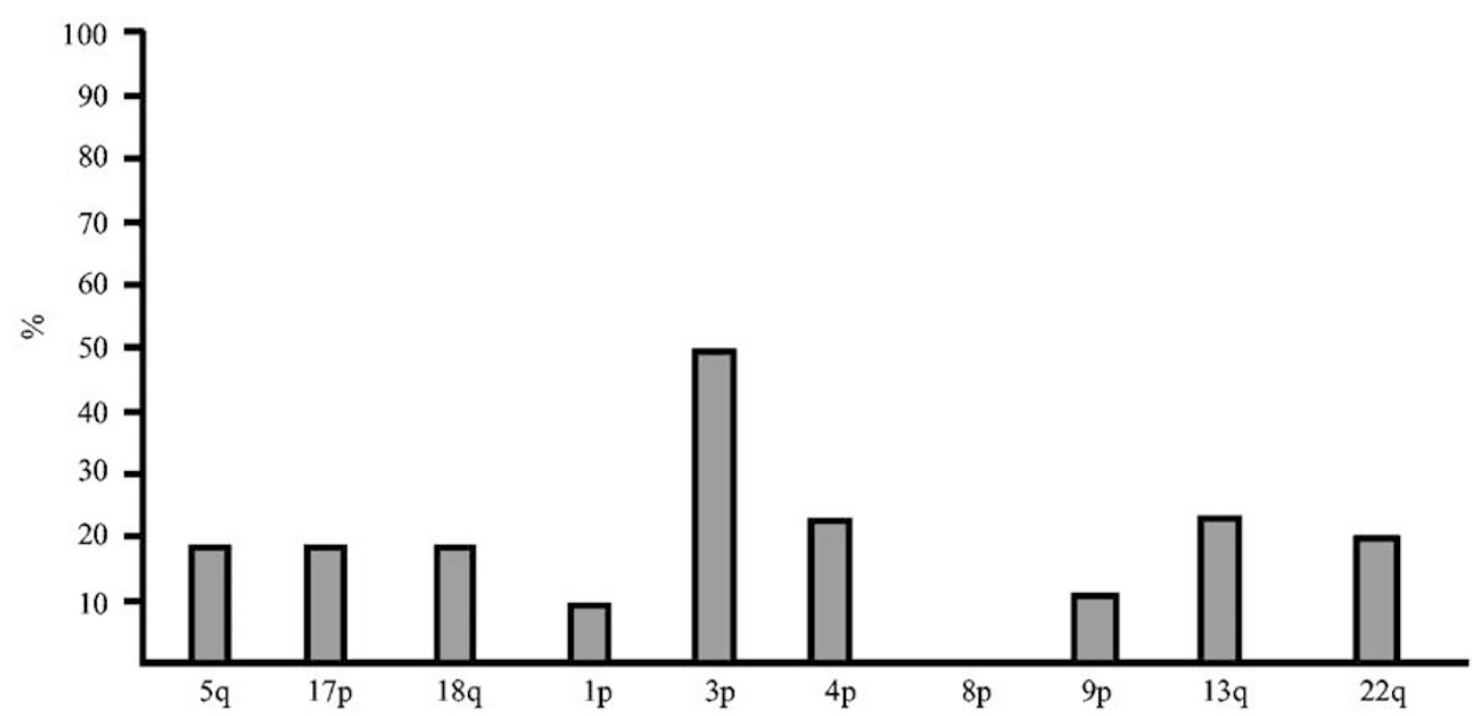

Figure 1 Frequencies of genetic alterations in the gastric phenotype of differentiated-type cancers.

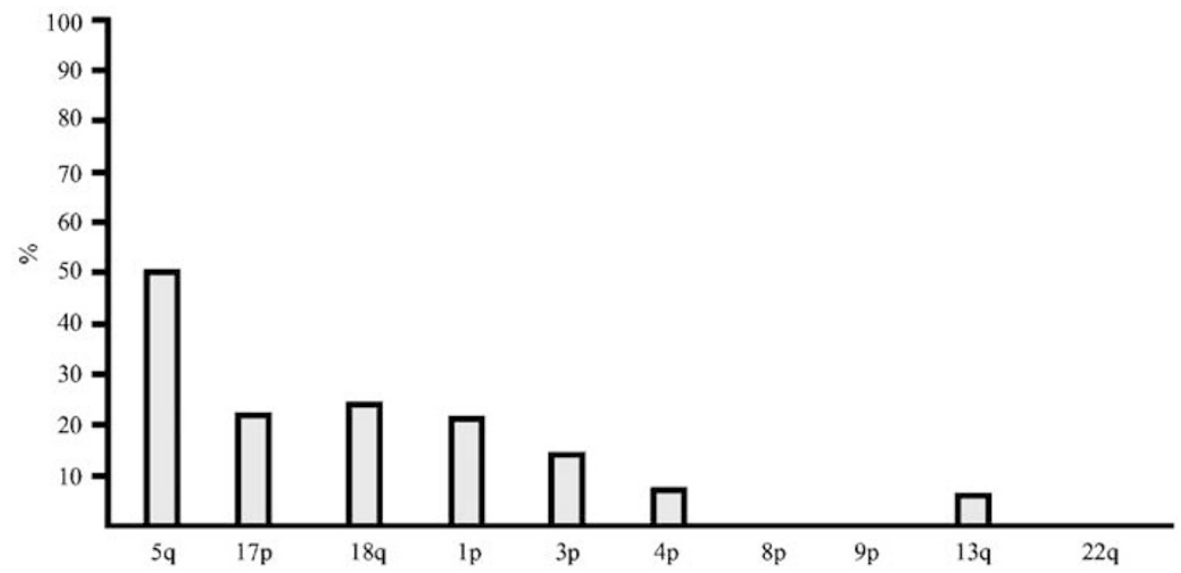

Figure 2 Frequencies of genetic alterations in the intestinal phenotype of differentiated-type cancers.

Table 3 Frequencies of genetic alterations in mixed-phenotype of differentiated-type cancers

\begin{tabular}{|c|c|c|c|c|c|c|c|c|c|c|c|}
\hline & Total & $5 q(\%)$ & $17 q(\%)$ & $18 q(\%)$ & $1 p(\%)$ & $3 p(\%)$ & $4 p(\%)$ & $8 p(\%)$ & $9 p(\%)$ & $13 p(\%)$ & $22 q(\%)$ \\
\hline $\mathrm{m}$ & 10 & $4 / 10(40)$ & 1/9 (11.1) & $3 / 10(30)$ & 1/10 (10) & $4 / 11(36.4)$ & 0/11 (0) & $1 / 11(10)$ & 1/11 (9.1) & 2/9 (22.2) & 1/10 (10) \\
\hline $\mathrm{sm}$ & 15 & $5 / 12(41.7)$ & $9 / 13(69.2)$ & $6 / 12(50)$ & $4 / 12(33.3)$ & $4 / 12(33.3)$ & $3 / 13(23.1)$ & $1 / 13(7.7)$ & $5 / 13(38.4)$ & $1 / 10(10)$ & $4 / 12(33.3)$ \\
\hline Total & 25 & $9 / 22(40.9)$ & $10 / 22(45.5)$ & $9 / 22(40.9)$ & $5 / 22(22.7)$ & $8 / 23(34.8)$ & $3 / 24(12.5)$ & 2/24 (8.3) & $6 / 24(25)$ & $3 / 19(15.8)$ & $5 / 22(22.7)$ \\
\hline
\end{tabular}

Table 4 Frequency of p53 overexpression in gastric-, intestinaland mixed phenotypes cancers

\begin{tabular}{lcccc}
\hline & $\begin{array}{c}\text { Gastric- } \\
\text { phenotype } \\
(\%)\end{array}$ & $\begin{array}{c}\text { Intestinal- } \\
\text { phenotype } \\
(\%)\end{array}$ & $\begin{array}{c}\text { Mixed- } \\
\text { phenotype } \\
(\%)\end{array}$ & $\begin{array}{c}\text { Unclassified } \\
\text { type }\end{array}$ \\
\hline $\mathrm{m}$ & $3 / 9(30)$ & $4 / 12(33.3)$ & $7 / 15(46.7)$ & 0 \\
$\mathrm{Sm}$ & $2 / 4(50)$ & $4 / 5(80)$ & $7 / 16(43.8)$ & $1 / 1$ \\
Total & $5 / 13(38.5)$ & $8 / 17(47.1)$ & $14 / 31(45.2)$ & $1 / 1$ \\
\hline
\end{tabular}

with the exceptional finding of a difference of histological type between intestinal- and gastricphenotypes $(P<0.05)$ (Table 1$)$. Allelic losses at each locus and tumor MSI were assessed for their association with tumor location, histology and tumor depth. Overall, no clinicopathological feature was significantly associated with allelic losses and MSI in intestinal-, gastric- and mixed-phenotypes. In addition, although follow-up for all patients operated on in this study was performed in our hospital, no recurrence or occurrence of metastasis has to date been identified. 
a

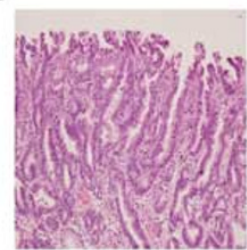

HE b

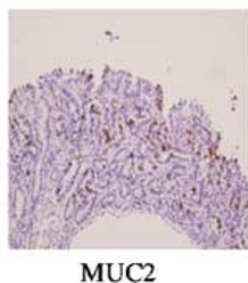

c

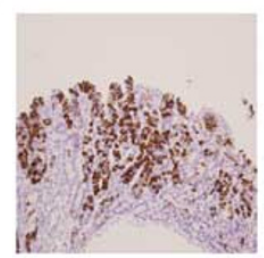

MUC5AC d

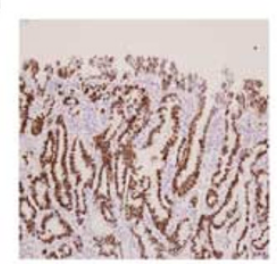

p53 stain

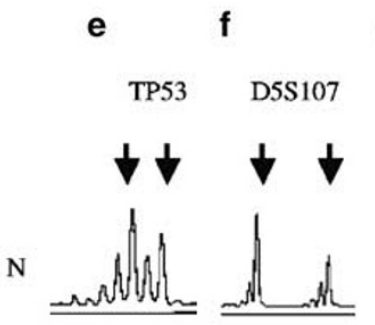

g

h

i D18S487 D22S274
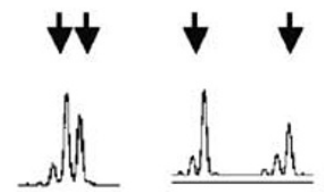

$\mathrm{T}$
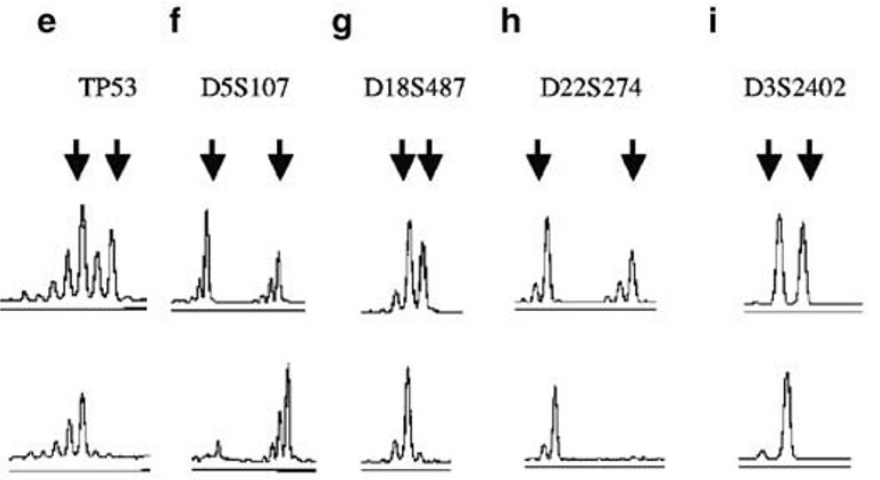

j D4S1601

k

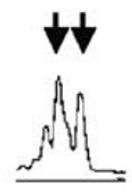

D9S1118

D13S162

Figure 3 Analysis of allelic losses at chromosomal loci on chromosomes 17p, 5q, 18q, 22q, 3p, 4p, 9p and 13p, and overexpression of p53 protein in mixed-phenotype cancers. (a) Histological features of differentiated-type adenocarcinoma. (b) and (c). Tumor cells strongly express MUC2 and MUC5AC. (d) Overexpression of p53 protein was observed. (e-l) Electropherogram demonstrating allelic losses at 17p, 5q, 18q, 22q, 3p, 4p, 9p and 13q chromosomal loci. Arrows show the two alleles for each chromosomal locus (N). One of the two alleles at each chromosomal locus was lost in e-l (T). N, normal; T, tumor.

a

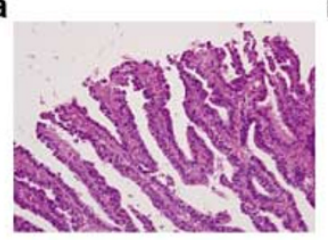

HE

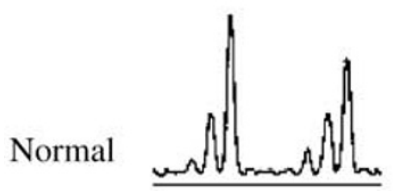

b

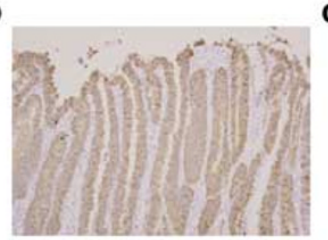

MUC2

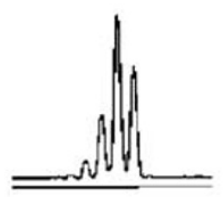

c

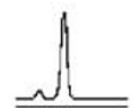

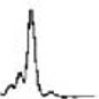
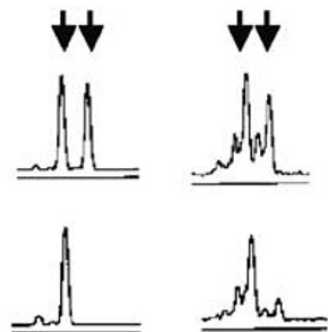

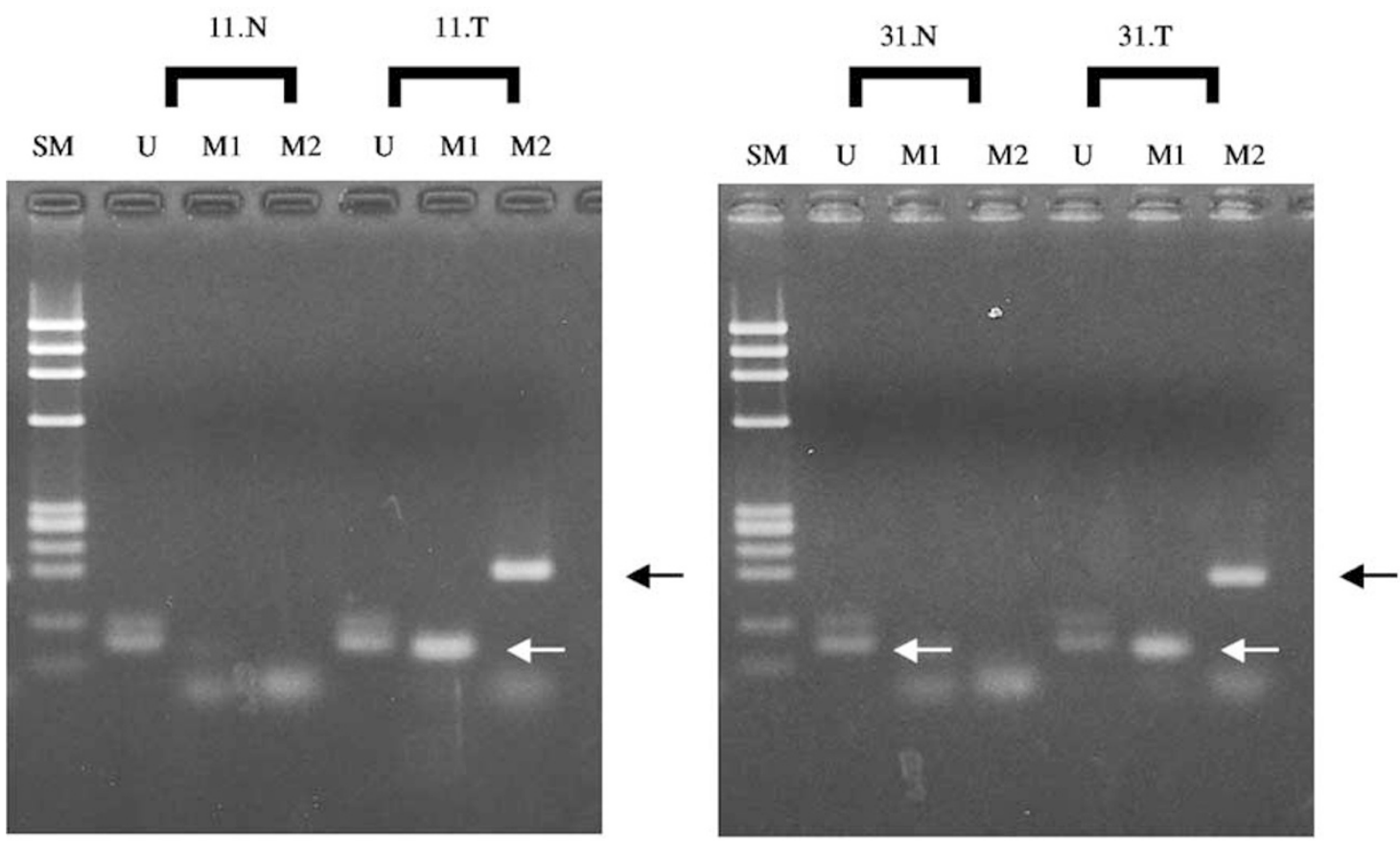

Figure 5 Example of the altered methylation status of the $h M L H 1$ promoter in gastric tumors detected by methylation-specific PCR. Two different primers were used to detect hMLH1 promoter hypermethylation status (M1 and M2). The presence of a visible PCR product in lane U indicates the presence of the unmethylated $h M L H 1$ promoter; the presence of product in lanes M1 and M2 indicates the presence of methylated hMLH1 promoter (arrowhead). Note that methylation of the hMLH1 promoter is found in normal cells (b-Lane M1) (arrowhead). SM, Size marker; N, Normal; T, Tumor.

gastric phenotype, were observed in 23 of 41 $(56.1 \%)$ differentiated-type gastric cancers. In addition, the frequency of the combined-type cancer, defined as mixed-phenotype cancer in this study, was six of $41(14.6 \%)$. These discrepancies may be due to the different criteria used for our definition of mucin. Endoh et $a l^{6,7}$ defined a foveolar-type cancer as a tumor mimicking gastric foveolar epithelium morphologically, and having intracytoplasmic mucin that is predominantly of the gastric foveolar epithelium type (50\% of neoplastic cells are positive for GOS stain or human gastric mucin). These criteria are different from those used in the current study. The current study used criteria to define mucins in colorectal carcinomas that were previously reported by Yao et al, ${ }^{17}$ given that this study showed that each mucin phenotype (small intestinal, large intestinal, gastric, mixed and unclassified types) has characteristic features in colorectal carcinomas. Whether the criteria used in this study truly is the cause of the difference between studies remains unclear. A tumor classified as a gastric phenotype according to the criteria used in this study may be reclassified as an unclassified type or a mixed-phenotype based on other criteria. On the other hand, a tumor classified as a gastric phenotype according to the other criteria may be reclassified as a mixed-phenotype based on the present criteria. As a result, we think that in the present study the frequency of mixed-phenotype was higher than that of other mucin phenotypes and that unclassifiedtype was rarely observed compared with previous studies. $^{6,7}$ In addition, different investigative approaches, the use of different antibodies and immunostaining procedures (eg the use of automatic vs manual staining), as well as tumor heterogeneity may at least partially explain the divergent findings. To establish the genetic profile of gastric differentiated-type adenocarcinoma categorized on the basis of mucin phenotype, a standardized method, such as immunohistochemical evaluation or sampling method, would be needed. Indeed, the most important reason for conflicting data between authors, as mentioned above, is that there is, as yet, no standardized method for categorizing differentiated gastric adenocarcinomas based on mucin phenotype. However, we believe that the immunostaining method (auto-immunostaining) and cutoff value of positive and negative that we used in this study are suitable for evaluating these cancers according to mucin profile because of their distinct genetic alterations.

We demonstrated that the frequency of $3 p$ allelic loss of gastric-phenotype is higher than that of intestinal-phenotype in differentiated-type cancers. Allelic loss at chromosome $3 p$ has been reported in a 
variety of malignancies including renal cell carcinoma, breast, lung, cervical and gastrointestinal cancers. ${ }^{24-27}$ In a study of gastric cancers, other investigators found that there was often a loss of heterozygosity (LOH) with allelic loss at D3S1234, which is known to be near 3p14.2. ${ }^{18}$ Recently, studies have suggested that the fragile histidine triad (FHIT) gene may be the specific target of 3p14.2 alterations in several types of human cancers. ${ }^{27,28}$ In the present study, D3S1234 was the polymorphic locus having the most frequent allelic loss. We suggest that allelic loss at $3 p$ may be a key event in the early carcinogenesis of gastric-phenotype cancers.

Allelic loss of chromosome $5 q$ was more frequently observed in intestinal-phenotype than in gastric-phenotype cancers. In addition, allelic losses at the other polymorphic chromosomal loci examined were rare. Others have reported that $5 \mathrm{q}$ allelic loss is found frequently in gastric adenomas. ${ }^{29}$ This finding suggests that $5 \mathrm{q}$ allelic loss may be an initial genetic event in gastric tumorigenesis. Vogelstein et al have reported that $\mathrm{LOH}$ on chromosome $5 \mathrm{q}$ is frequently associated with colorectal adenomas and adenocarcinomas (most of which are well-differentiated adenocarcinomas). ${ }^{30}$ It is possible that welldifferentiated intestinal-phenotype gastric cancers develop through genetic alterations in a manner similar to those observed in colorectal carcinomas that express the intestinal mucin phenotype. However, in Japan, it is known that gastric adenoma is not a precursor lesion of early gastric carcinoma. ${ }^{31}$ Thus, we believe that the intestinal phenotype of early gastric cancers does not derive from gastric adenoma. We suggest that intestinal-phenotype cancers have genetic profiles that set the cells on different pathways from those leading to the development of gastric adenomas, although both tumors may share common genetic alterations in their initial tumorigenesis. ${ }^{32}$

In the present study, mixed-phenotype early gastric cancers were classified into two distinct genetic pathways, LOH and MSI. $3 p$, $5 q$ and $18 q$ allelic losses were frequently found in intramucosal mixed-phenotype carcinomas. Our data suggest that although allelic losses at $3 p$ and $5 q$ are early genetic alterations common to either gastric- or intestinalphenotype cancers, $18 \mathrm{q}$ allelic loss may be an early and distinct genetic alteration that occurs only in mixed-phenotype differentiated-type gastric cancers. However, other studies have suggested that $18 \mathrm{q}$ allelic loss appears later: it is infrequent in intramucosal or minimally invasive submucosal carcinomas, but increases with progression to massively invasive submucosal carcinomas and more advanced carcinomas. ${ }^{32-34}$ Our results, however, indicate that allelic loss at $18 \mathrm{q}$ may be an essential early event in the carcinogenesis of mixedphenotype differentiated-type gastric cancers.

We also demonstrated a high frequency of allelic losses at $17 p, 1 p$ and $9 p$ in gastric submucosal cancers of mixed phenotype, suggesting that these genetic alterations, in particular $17 \mathrm{p}$ allelic loss, play an important role in submucosal invasion by mixed-phenotype cancers. Chromosome $17 \mathrm{p}$ is known to harbor the p53 tumor suppressor gene. Allelic loss at the TP53 microsatellite marker, which we used as a primer set, represents $\mathrm{LOH}$ of the $p 53$ gene. Although loss or inactivation of p53 function is well documented in a large number of human cancers, including gastric cancers, ${ }^{35-37}$ the timing of its dysfunction in carcinogenesis is not fully understood. Ranzani et $a l^{38}$ reported that p53 gene alterations (found also in precancerous dysplastic lesions) were frequent in intramucosal tumors. This finding is not consistent with our results, which demonstrate infrequent allelic loss of $17 \mathrm{p}$ in intramucosal cancers of mixed phenotype. However, our present study agrees with our previous study, in that no $17 \mathrm{p}$ allelic loss was found in intramucosal cancers. ${ }^{3}$ In addition, our finding that $17 \mathrm{p}$ allelic loss is frequently detected in submucosal cancers of mixed phenotype supports our previous study that suggests allelic loss of $p 53$ gene in differentiated-type gastric cancer is associated with the development of submucosal cancer. ${ }^{3}$

We found in this study that overexpression of the p53 protein is common in differentiated-type gastric cancers. ${ }^{4,39}$ However, a previous report indicated that p53 overexpression occurs in the later stages of differentiated-type gastric cancers. ${ }^{40}$ Tahara ${ }^{41}$ indicated that $p 53$ gene alterations are found in intestinal metaplasia and gastric adenomas. However, we did not detect any p53 overexpression either in intestinal metaplasia surrounding the gastric cancer tissue or in gastric adenomas (data not shown). We suspect that overexpression of p53 plays a fundamental role in the carcinogenesis of early differentiated adenocarcinomas of the stomach.

Alternative modes of gene inactivation during the development of cancer include an epigenetic process that is marked by promoter region hypermethylation and associated with transcriptional loss, which has been demonstrated for tumor suppressor genes and DNA mismatch repair (MMR) genes. ${ }^{22,23}$ MSI is thought to be due to inactivation of MMR genes. ${ }^{22}$ In the present study, MSI was detected in six of 31 mixed-phenotype early gastric cancers. However, no MSI was found in gastric- and intestinal-phenotypes cancers. In addition, hypermethylation of the hMLH1 promoter was found in five of six tumors with high MSI. Endoh et $a l,{ }^{6}$ in contrast, found a high frequency of MSI and $h M L H 1$ promoter hypermethylation in both the foveolarand combined-type tumors (gastric and mixed phenotypes, respectively, by our classification), but not in the intestinal-type tumors. However, it may prove difficult to explain this discrepancy. As mentioned above, we believe that at least one of the reasons may be related to the definition of mucin 


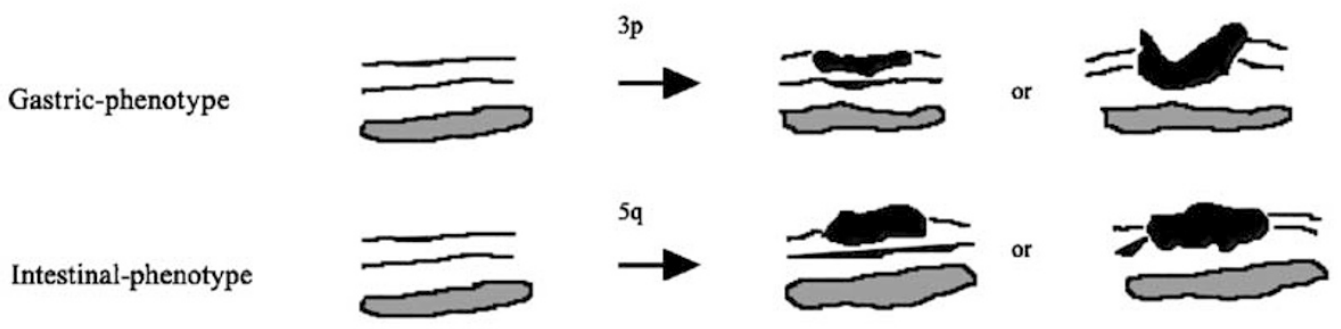

Common genetic alterations: Overexpression of p53 gene
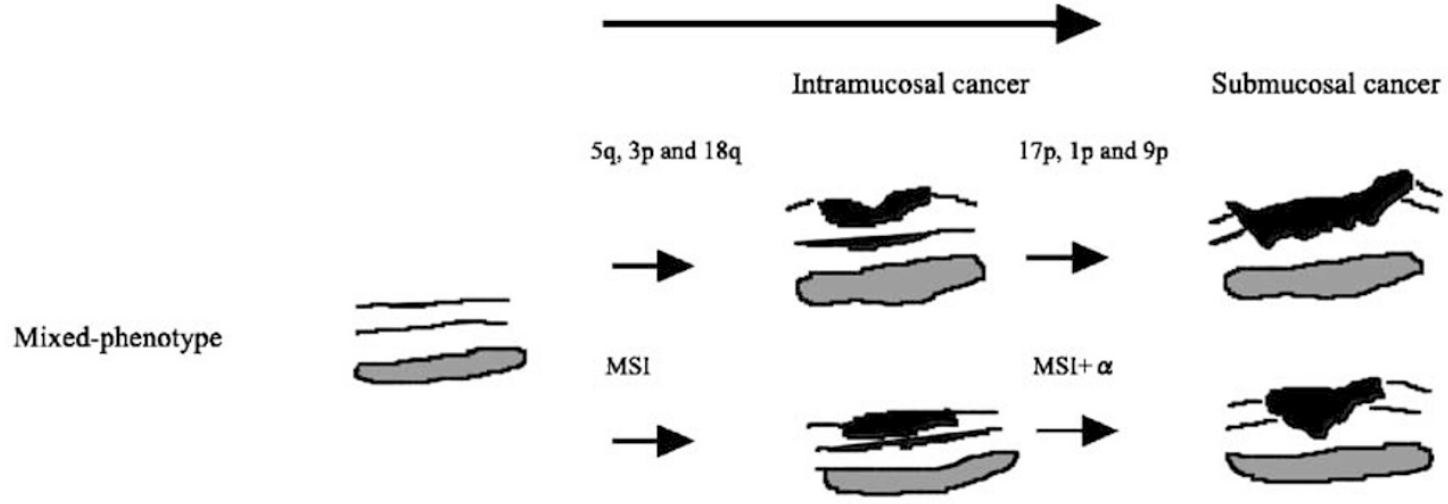

Figure 6 A new hypothesis of gastric tumor progression as defined by mucin phenotype. The solid line represents the main pathway of tumor progression. $3 p$ and $5 q$ allelic losses may be involved in the development of gastric- and intestinal-phenotype cancers, respectively. On the other hand, two distinct genetic pathways play a major role in the progression of mixed-phenotype cancers: $\mathrm{LOH}$ and MSI. Although allelic losses of 5q, 3p and 18q may be initial steps in the carcinogenesis of intramucosal cancer of mixed phenotype, allelic losses of 17p, 1p and 9p are closely associated with submucosal invasion of mixed phenotype cancers. MSI is detected in mixedphenotype cancers only. Overexpression of p53 protein is a common event in differentiated-type early gastric cancers. MSI, microsatellite instability.

phenotypes for the tumor cells. Our study suggests that the occurrence of MSI is associated with a specific histomorphological type of gastric cancer (ie, the mucin phenotype). Considering the high frequency of p53 overexpression in MSI-type tumors in the present study, this type of tumor may acquire a highly malignant potential. However, in general, overexpression of the p53 protein is inversely correlated with the frequency of MSI. ${ }^{42}$ Furthermore, in sporadic colorectal cancers, MSI positivity is associated with a favorable prognosis. ${ }^{43}$ However, in the present study, although there was no significant difference in the frequency of p53 overexpression between MSI + and MSI- tumors of the mixed type, the frequency of p53 overexpression in MSI + tumors ( $4 / 6$ cases) was higher than that in MSI- tumors (10/25 cases). We believe that p53 overexpression may be associated with the development of MSI + tumors, though conflicting data have been reported. ${ }^{16}$

In summary, we propose that the cellular mucin phenotypes of differentiated-type adenocarcinomas of the stomach are dependent on distinct genetic profiles that result in different metabolic pathways (gastric-, intestinal- and mixed-phenotype). A novel carcinogenesis for early differentiated-type adeno- carcinomas that relies on their mucin phenotypes is illustrated in Figure 6. 3p allelic loss may be an early event in gastric-phenotype cancers, whereas $5 \mathrm{q}$ allelic loss is associated with the development of intestinal-type cancers. Although other gene(s) may also be responsible for invasion of cancer cells in both mucin phenotype cancers, further investigation is needed. On the other hand, the genetic profiles of mixed-phenotype cancers were composed of two distinct genetic types: the LOH and MSI types. Whereas the former type involves $5 q, 3 p$ and $18 q$ allelic losses in intramucosal cancers of mixed phenotype, $17 p, 1 p$ and $9 p$ allelic losses play a major role in the submucosal invasion of mixed phenotype cancers. MSI is closely associated with tumorigenesis of a subset of differentiated-type cancers. Overexpression of p53 protein is a common event in early differentiated-type adenocarcinomas, regardless of the mucin phenotypes. Finally, in the present study, only one of 62 tumors was of the unclassified type, and this tumor showed numerous genetic alterations. Little is known of the distinct pathways involved in the pathogenesis of the unclassified phenotype of gastric cancer. Further studies will be necessary to answer this question. 


\section{References}

1 Lauren P. The two histological main types of gastric carcinomas: diffuse and so-called intestinal-type carcinoma. Acta Pathol Microbiol Immunol Scand 1965;64:31-49.

2 Nakamura K, Sugano H, Takagi K. Carcinoma of the stomach in incipient phase: its histogenesis and histological appearances. GANN 1968;59:251-258.

3 Sugai T, Habano W, Nakamura S, et al. Correlation of histological morphology and tumor stage with molecular genetic analysis using microdissection in gastric carcinomas. Diagn Mol Pathol 1998;7:235-240.

4 Sugai T, Nakamura S, Uesugi N, et al. Role of DNA aneuploidy, overexpression of p53 gene product and cellular proliferation in the progression of gastric cancer. Cytometry 1999;38:111-117.

5 Fiocca R, Luinetti O, Villani L, et al. Molecular mechanisms involved in the pathogenesis of gastric carcinoma: interactions between genetic alterations, cellular phenotype and cancer histotype. Hepatogastroenterology 2001;48:1523-1530.

6 Endoh Y, Tamura G, Ajioka Y, et al. Frequent hypermethylation of the hMLH1 gene promoter in differentiated-type tumors of the stomach with the gastric foveolar phenotype. Am J Pathol 2000;157: 717-722.

7 Endoh Y, Sakata K, Tamura G, et al. Cellular phenotypes of differentiated-type adenocarcinomas and precancerous lesions of the stomach are dependent on the genetic pathways. J Pathol 2000;191:257-263.

8 Vogelstein B, Fearon ER, Hamilton SR, et al. Genetic alterations during colorectal tumor development. N Eng J Med 1988;319:525-532.

9 Lengauer C, Kinzler KW, Vogelstein B. Genetic instabilities in human cancers. Nature 1998;396: 643-649.

10 Thibodeau SN, Bren G, Schaid D. Microsatellite instability in cancer of the proximal colon. Science 1993;260:816-819.

11 Japanese Research Society for Gastric Cancer. The General Rules for the Gastric Cancer Study. 12th edn. Tokyo: Kanehara-shuppan, 1993, pp 64-89.

12 Schlemper RJ, Itabashi M, Kato Y, et al. Differences in diagnostic criteria for gastric carcinoma between Japanese and western pathologists. Lancet 1997;349: 1725-1729.

13 Lewin KJ, Appelman HD. Tumors of the Esophagus and Stomach: Carcinoma of the stomach. Washington DC: AFIP, 1995, pp 267-276.

14 Sugai T, Habano W, Nakamura S, et al. A unique method for mutation analysis of tumor suppressor genes in colorectal carcinomas using a crypt isolation technique. Arch Pathol Lab Med 2000;124:382-386.

15 Hsu C, Huang CL, Hsu HC, et al. HER-2/neu overexpression is rare in hepatocellular carcinoma and not predictive of anti-HER-2/neu regulation of cell growth and chemosensitivity. Cancer 2002;94:415-420.

16 Ohmura K, Tamura G, Endoh Y, et al. Microsatellite alterations in differentiated-type adenocarcinomas and precancerous lesions of the stomach with special reference to cellular phenotype. Hum Pathol 2000; 31:1031-1035.

17 Yao T, Tsutsumi S, Akaiwa Y, et al. Phenotypic expression of colorectal adenocarcinomas with reference to tumor development and biological behavior. Jpn J cancer Res 2001;92:755-761.
18 Yustein AS, Harper JC, Petroni GR, et al. Allelotype of gastric adenocarcinoma. Cancer Res 1999;59: 1437-1441.

19 Habano W, Sugai T, Nakamura S, et al. A novel method for gene analysis of colorectal carcinomas using a crypt isolation technique. Lab Invest 1996;74: 933-940.

20 Sugai T, Habano W, Nakamura S, et al. Genetic alterations in DNA diploid, aneuploid and multiploid colorectal carcinomas identified by the crypt isolation technique. Int J Cancer 2000;88:614-619.

21 Halling KC, Harper J, Moskaluk CA, et al. Origin of microsatellite instability in gastric cancer. Am J Pathol 1999;155:205-211.

22 Herman JG, Umar A, Polyak K, et al. Incidence and functional consequences of hMLH1 promoter hypermethylation in colorectal carcinoma. Proc Natl Acad Sci USA 1998;95:6870-6875.

23 Salvesen HB, MacDonald N, Ryan A, et al. Methylation of hMLH1 in a population-based series of endometrial carcinomas. Clin Cancer Res 2000;6:3607-3613.

24 Sukosd F, Kuroda N, Beothe T, et al. Deletion of chromosome 3p14.2-p25 Involving the VHL and FHIT Genes in Conventional Renal Cell Carcinoma. Cancer Res 2003;63:455-457.

25 Yang Q, Nakamura M, Nakamura Y, et al. Two-hit inactivation of FHIT by loss of heterozygosity and hypermethylation in breast cancer. Clin Cancer Res 2002;8:2890-2893.

26 Takebayashi Y, Nakayama K, Kanzaki A, et al. Loss of heterozygosity of nucleotide excision repair factors in sporadic ovarian, colon and lung carcinomas: implication for their roles of carcinogenesis in human solid tumors. Cancer Lett 2001;174:115-125.

27 Noguchi T, Muller W, Wirtz HC, et al. FHIT gene in gastric cancer: association with tumour progression and prognosis. J Pathol 1999;188:378-381.

28 Baffa R, Veronese ML, Santoro R, et al. Loss of FHIT expression in gastric carcinoma. Cancer Res 1998; 58:4708-4714.

29 Neuman WL, Wasylyshyn ML, Jacoby R, et al. Evidence for a common molecular pathogenesis in colorectal, gastric, and pancreatic cancer. Genes Chromosomes Cancer 1991;3:468-473.

30 Fearon ER, Vogelstein B. A genetic model for colorectal tumorgenesis. Cell 1990;61:759-767.

31 Kamiya T, Morishita T, Asakura H, et al. Long-term follow-up study on gastric adenoma and its relation to gastric protruded carcinoma. Cancer 1982;50: 2496-2503.

32 Sano T, Tsujino T, Yoshida K, et al. Frequent loss of heterozygosity on chromosomes 1q, 5q, and $17 p$ in human gastric carcinomas. Cancer Res 1991;51: 2926-2931.

33 Inoue $\mathrm{T}$, Uchino $\mathrm{S}$, Shiraishi $\mathrm{N}$, et al. Loss of heterozygosity on chromosome $18 \mathrm{q}$ in cohesivetype gastric cancer is associated with tumor progression and poor prognosis. Clin Cancer Res 1998;4: 973-977.

34 Nishizuka S, Tamura G, Terashima M, et al. Loss of heterozygosity during the development and progression of differentiated adenocarcinoma of the stomach. J Pathol 1998;185:38-43.

35 Lim BH, Soong R, Grieu F, et al. p53 accumulation and mutation are prognostic indicators of poor survival in human gastric carcinoma. Int J Cancer 1996;69: 200-204. 
36 Soong R, Robbins PD, Dix BR, et al. Concordance between p53 protein overexpression and gene mutation in a large series of common human carcinomas. Hum Pathol 1996;27:1050-1055.

37 Tamura G. Molecular pathogenesis of adenoma and differentiated adenocarcinoma of the stomach. Pathol Int 1996;46:834-841.

38 Ranzani GN, Luinetti O, Padovan LS, et al. p53 gene mutations and protein nuclear accumulation are early events in intestinal type gastric cancer but late events in diffuse type. Cancer Epidemiol Biomarkers Prev 1995;4:223-231.

39 Wu MS, Shun CT, Lee WC, et al. Overexpression of p53 in different subtypes of intestinal metaplasia and gastric cancer. Br J Cancer 1998;78:971-973.
40 Maehara Y, Tomoda M, Hasuda S, et al. Prognostic value of p53 protein expression for patients with gastric cancer-a multivariate analysis. Br J Cancer 1999;79:1255-1261.

41 Tahara E. Molecular mechanism of stomach carcinogenesis. J Cancer Res Clin Oncol 1993;119: 265-272.

42 Habano W, Sugai T, Nakamura S, et al. Microsatellite instability and mutation of mitochondrial and nuclear DNA in gastric carcinoma. Gastroenterology 2000;118:835-841.

43 Hemminki A, Mecklin JP, Jarvinen $\mathrm{H}$, et al. Microsatellite instability is a favorable prognostic indicator in patients with colorectal cancer receiving chemotherapy. Gastroenterology 2000;119:921-928. 